\section{Similar Solutions for Boundary Layers in Constant-Temperature Magneto-Gasdynamic Channel Flow}

Jack L. Kerrebrock

Daniel and Florence Guggenheim Jet Propulsion Center, California Institute of Technology, Pasadena, Calif.

September 17, 1959

O NE OF THE PROBLEMS that must be faced in the development of plasma accelerators is the growth of boundary layers upon the accelerator walls. The boundary-layer effects may be important not only from a heat-transfer standpoint, but because of the displacement effect on the main flow. For the steady-flow accelerator utilizing crossed electric and magnetic fields, such as that discussed by Resler and Sears, a very interesting situation may develop upon the walls that are perpendicular to the current flow. Since the wall must generally be cool, there is a tendency for the plasma conductivity near the wall to be lower than that in the relatively hot free stream. Consequently, the Joule heating associated with the continuous current will be highest in the neighborhood of the wall. This increased heating may produce an abnormal thermal boundary layer and, quite possibly, a severe heat-transfer condition.

The boundary-layer development may be analyzed, utilizing as free-stream conditions the simple, constant-temperature channel-flow solutions presented in a previous note. ${ }^{1}$ It was shown in reference 1 that, if the free-stream velocity varies as a power of the streamwise coordinate, the pressure, current density, and flow area are also proportional to powers of the coordinate, as is the required magnetic-field strength, except for an additive constant. This simple behavior suggests that similar solutions, analogous to those of Falkner and Skan for the incompressible boundary layer, may exist for the boundary layer on the electrodes. It will be shown in the following paragraphs that such solutions do in fact exist, if the Mach Number is small. By utilizing the concept of local similarity, it may be possible to extend the results to nonzero Mach Numbers.

The usual boundary-layer equations must be modified by the inclusion of the electromagnetic body force in the momentum equation, and the electronic conduction and Joule heating in the energy equation. Thus, if $j, \sigma$, and $B$ denote the current density, electrical conductivity, and magnetic-field strength, the equations are:

continuity, $[\partial(\rho u) / \partial x]+[\partial(\rho v) / \partial y]=0$

momentum, $\rho[u(\partial u / \partial x)+v(\partial u / \partial y)]=$

$$
(\partial / \partial y)[\mu(\partial u / \partial y)]-\dot{(d p / d x)+j B}
$$

energy, $\quad \rho c_{p}[u(\partial T / \partial x)+v(\partial T / \partial y)]=$

$$
\begin{array}{r}
(\partial / \partial y)[\lambda(\partial T / \partial y)+(5 / 2)(k / e) j T]+ \\
\mu[(\partial u / \partial y)]^{2}+u(d p / d x)+\left(j^{2} / \sigma\right)
\end{array}
$$

and state,

$$
p=\rho R T
$$

where $k$ and $e$ are Boltzmann's constant and the magnitude of the electronic charge, and the other notation is conventional.

It is assumed that the magnetic Reynolds Number is sufficiently low so that $B$ may be regarded as a prescribed function of $x$. The current density is also assumed to be a function only of $x$, the form of which is determined by the free-stream flow. Thus, the electrical resistance of the boundary layer is considered a second-order correction to the free-stream resistance, just as the displacement thickness of a boundary layer is considered a small correction to the body contour.

In the channel-flow approximation, the momentum and energy equations for the free-stream flow are

and

$$
\rho_{\infty} u_{\infty}\left(d u_{\infty} / d x\right)=-(d p / d x)+j B
$$

$$
u_{\infty}(d p / d x)=-\left(j^{2} / \sigma_{\infty}\right)
$$

With these relations, the boundary-layer momentum and energy equations become

$$
\begin{aligned}
\rho[u(\partial u / \partial x)+v(\partial u / \partial y)]=(\partial / \partial y)[\mu(\partial u / \partial y)]+ \\
\rho_{\infty} u_{\infty}\left(d u_{\infty} / d x\right)
\end{aligned}
$$

and

$\rho c_{p}[u(\partial T / \partial x)+v(\partial T / \partial y)]=$

$$
\begin{array}{r}
(\partial / \partial y)[\lambda(\partial T / \partial y)+(5 k / 2 e) j T]+ \\
\mu(\partial u / \partial y)^{2}+\left(j^{2} / \sigma_{\infty}\right)\left[\left(\sigma_{\infty} / \sigma\right)-\left(u / u_{\infty}\right)\right]
\end{array}
$$

Since the free-stream pressure varies while the free-stream temperature is constant, the following modification of the Stewartson transformation ${ }^{3,4}$

$$
\bar{y}=\int_{0}^{y}\left(\rho / \rho_{0}\right) d y, \quad \bar{x}=\int_{0}^{x}\left(p / p_{0}\right) d x
$$

where the subscript 0 denotes values in the free stream at a reference point is convenient. It gives $\vec{u}=u$ and $\bar{v}=\left(p_{0} / p\right)\left[\left(\rho v / \rho_{0}\right)\right.$ $+u(\partial \bar{y} / \partial x)]$, where if $\mu$ and $\lambda$ are proportional to $T$, as for Maxwellian molecules, $\bar{u}$ and $\bar{v}$ satisfy the transformed equations:

$$
\begin{gathered}
(\partial \bar{u} / \partial \bar{x})+(\partial \bar{v} / \partial \bar{y})=0 \\
\bar{u}(\partial \bar{u} / \partial \bar{x})+\bar{v}(\partial \bar{u} / \partial \bar{y})=\nu_{0}\left(\partial^{2} \bar{u} / \partial \bar{y}^{2}\right)+\theta\left[\bar{u}_{\infty}\left(d \bar{u}_{\infty} / d \bar{x}\right)\right]
\end{gathered}
$$

The dimensionless temperature, $\theta \equiv T / T_{0}$, satisfies

$$
\begin{aligned}
& \bar{u}(\partial \theta / \partial \bar{x})+ \bar{v}(\partial \theta / \partial \bar{y})=\left(\nu_{0} / \operatorname{Pr}\right)\left(\partial^{2} \theta / \partial \bar{y}^{2}\right)+ \\
&\left(\nu_{0} / c_{p} T_{0}\right)(\partial \bar{u} / \partial \bar{y})^{2}+\left(5 k j / 2 e c_{p} \rho_{0}\right)\left(p_{0} / p\right)(\partial \theta / \partial \bar{y})+ \\
&\left(j^{2} / c_{p} T_{0} \sigma_{0} \rho_{\infty}\right)\left(p_{0} / p\right) \theta\left[\left(\sigma_{\infty} / \sigma\right)-\left(\bar{u} / \bar{u}_{\infty}\right)\right]
\end{aligned}
$$

A simple solution for channel flow with constant temperature, conductivity, and electric field was given in reference 1 . It was demonstrated that, if the velocity is of the form $u=\alpha x^{n}$, the current and pressure are given by

$$
j=\left(\sigma_{0} R T_{0} E / \alpha^{2} n\right)(5 n-1) x^{-2 n}
$$

and

$$
p=\left[\sigma_{0}\left(R T_{0} E\right)^{2} / \alpha^{5}\right]\left[(5 n-1) / n^{2}\right] x^{1-5 n}
$$

where $E$ is the constant electric field.

With this expression for the pressure, the transformation from $x$ to $\bar{x}$ becomes

$$
\bar{x}=K x^{2-5 n}
$$

where

$$
K \equiv\left[\sigma_{0}\left(R T_{0} E\right)^{2} / n^{2} \alpha^{5} p_{0}\right][(5 n-1) /(2-5 n)]
$$

and $n$ must be less than $2 / 5$.

Because of the simple power relation between $x$ and $\bar{x}$, the transformed velocity, $\bar{u}_{\infty}$, varies as a simple power of $\bar{x}$. If we take $\bar{u}_{\infty}=\beta \bar{x}^{m}$, it follows that $\beta=\alpha / K^{m}$ and $m=n /(2-5 n)$. With the transformed free-stream velocity in this form, the transformed momentum equation is that solved by Falkner and Skan, except that the factor $\theta$ has replaced unity in the last term.

Following Schlichting, ${ }^{5}$ we define the coordinate $\eta$ by

$$
\eta \equiv \tilde{y} \sqrt{[(m+1) / 2]\left(\beta / \nu_{0}\right)} \bar{x}^{(m-1) / 2}
$$

and take the stream function as

$$
\psi=\sqrt{\left[2 \nu_{0} \beta /(m+1)\right] \bar{x}^{(m+1) / 2} f(\eta)}
$$

whence $\bar{u}=\bar{u}_{\infty} f^{\prime}(\eta)$, the prime denoting differentiation,

$$
\begin{aligned}
& \bar{v}=-\sqrt{\left[2 \nu_{0} \beta /(m+1)\right]} \times \\
& {[(m+1) / 2] \bar{x}^{(m-1) / 2}\left\{f+[(m-1) /(m+1)] \eta f^{\prime}\right\}}
\end{aligned}
$$

and the momentum equation reduces to

$$
f^{\prime \prime \prime}+f f^{\prime \prime}+[n /(1-2 n)]\left[\theta-\left(f^{\prime}\right)^{2}\right]=0
$$

By utilizing the expressions for the current and pressure given by the channel flow, the energy equation may be put in the form

$$
\begin{array}{r}
(1 / P r)\left(\partial^{2} \theta / \partial \eta^{2}\right)+f(\partial \theta / \partial \eta)= \\
{[2 \bar{x} /(m+1)] f^{\prime}(\partial \theta / \partial \bar{x})-(\gamma-1) \bar{M}_{\infty}{ }^{2}\left(f^{\prime \prime}\right)^{2}-} \\
{[(\gamma-1) / \gamma][(5 n-1) /(1-2 n)]^{1 / 2}\left(5 k T_{0} / 2 e\right)\left(\sigma_{0} / \mu_{0} R T_{0}\right)^{1 / 2} \times} \\
(\partial \theta / \partial \eta)-[(\gamma-1) / \gamma[(5 n-1) /(1-2 n)] \times \\
\theta\left[\left(\sigma_{\infty} / \sigma\right)-f^{\prime}\right]
\end{array}
$$


$\bar{x}$ does not occur in Eq. (12) and occurs in Eq. (13) only in the first and second terms on the right. If the Mach Number, which depends on $\bar{x}$, is small and the boundary conditions are independent of $\bar{x}$, solutions of Eqs. (12) and (13) exist such that $f$ and $\theta$ depend only on $\eta$, since then the term proportional to $\partial \theta / \partial \bar{x}$ is zero. Thus, similar solutions exist for the thermal and viscous boundary layers if $\bar{M}_{\infty}{ }^{2}$ is small and the wall temperature is constant.

It is worth noting that both the channel-flow solution and the boundary-layer transformation can be extended to include variations of the conductivity and electric field as powers of $x$. The momentum equation retains the form given above, as does the energy equation, except that the free-stream conductivity, which occurs in the coefficient of $\partial \theta / \partial \eta$, becomes a function of $\bar{x}$. Thus, exact similarity no longer exists for small $\bar{M}_{\infty}{ }^{2}$ if $\sigma_{\infty}$ varies with $x$

Nevertheless, for many practical cases, the similarity may be nearly exact because the electronic conduction term is small compared to the Joule-heating term. As an example, for helium at $3,000^{\circ} \mathrm{K}$., seeded to a conductivity of $100 \mathrm{mho} / \mathrm{m}$, the coefficient of $\partial \theta / \partial \eta$ is 0.05 , while that of $\theta\left(\sigma_{\infty} / \sigma-f^{\prime}\right)$ is 0.2 , if $n=1 / 4$.

The range of $n$ which is physically interesting is limited by the channel-flow solution to positive values greater than $1 / 5$, while the present transformation from $x$ to $\bar{x}$ limits it to values below $2 / 5$. Over the range of $n$ from $1 / 5$ to $2 / 5, m$ varies from $1 / 5$ to $\infty$, so that boundary-layer flows with a very wide range of characteristics are described.

\section{REFERENCES}

' Kerrebrock, J. L., and Marble, F. E., Constant-Temperature Magneto Gasdynamic Channel Flow, Readers' Forum, Journal of the Aero/Space Sciences, Vol. 27, No. 1, p. 78, January, 1960.

${ }^{2}$ Falkner, V. M., and Skan, S. W., Some Approximate Solutions of the Botindary Layer Eqtations, Phil. Mag., Vol. 12, p. 865, 1931.

${ }^{3}$ Stewartson, K., Correlated Incompressible and Compressible Boundary Layers, J. Royal Society, A, Vol. 200, p. 84, 1931.

Cohen, C. B., and Reshotko, Eli, Similar Solutions for the Compressible Laminar Boundary Layer zeith Heat Transfer and Pressure Gradient. NACA TN 3325, February, 1955.

s Schlichting, H., Boundary Layer Theory, p. 128, McGraw-Hill Book Company, Inc. New York, 1955. 